\title{
Highly selective charge-guided ion transport through a hybrid membrane consisting of anionic graphene oxide and cationic hydroxide nanosheet superlattice units
}

\author{
Pengzhan Sun ${ }^{1,2}$, Renzhi $\mathrm{Ma}^{2}$, Wei $\mathrm{Ma}^{2}$, Jinghua $\mathrm{Wu}^{2}$, Kunlin Wang ${ }^{1}$, Takayoshi Sasaki ${ }^{2}$ and Hongwei Zhu ${ }^{1,3}$ \\ The development of graphene-based functional membranes with the ability to effectively filter and separate molecules or ions in \\ solutions based on a simple criterion (for example, the size or charge of solutes) is crucial for various engineering-relevant \\ applications, ranging from wastewater purification and reuse to chemical refinement. Here, we report a hybrid membrane \\ consisting of anionic graphene oxide (GO) and cationic Co-Al (or Mg-Al) layered double hydroxide (LDH) nanosheet (NS) \\ superlattice units for high selectivity charge-guided ion transport. The hybrid membrane possesses a series of characteristics, \\ including being easy to access, mechanically robust, freestanding, flexible and semitransparent as well as having a large area. \\ The interlayer spacing of the hybrid membrane is insensitive to humidity variations, ensuring the structural stability in \\ solution-based mass transport applications. The concentration gradient-driven ion transmembrane diffusion experiments show \\ that the cations bearing various valences can be effectively separated strictly according to their charges, independent of the \\ cationic and charge-balancing anionic species. The relative selectivity of the hybrid membranes toward monovalent and trivalent \\ cations is as high as 30 , which is not achievable by GO multilayer stacks, LDH-NS multilayer stacks or their bulk-stratified \\ membranes, indicating that a synergistic effect originating from the molecular-level heteroassembly of GO and LDH-NS has \\ a dominant role in the high-performance charge-guided ion filtration and separation processes. These excellent properties of \\ GO/LDH-NS hybrid membranes make them promising candidates in diverse applications, ranging from wastewater treatment and \\ reuse and chemical refinement to biomimetic selective ion transport.
}

NPG Asia Materials (2016) 8, e259; doi:10.1038/am.2016.38; published online 1 April 2016

\section{INTRODUCTION}

The ever-deepening crisis of fresh water shortage and contamination worldwide and the increasingly high demand for chemical refinement have accelerated the pursuit of membrane-based filtration and separation technologies with the merits of being cost-effective, low in energy consumption, easy to operate and, more importantly, not entailing the use of chemicals and minimizing impact on the environment. ${ }^{1-3}$ For this purpose, the development of membranes with novel separation functions is a key objective.

Recently, the rapid advancement of nanostructured materials, especially carbon nanomaterials (for example, carbon nanotubes, graphene and its derivatives), has opened new avenues for fabricating next-generation membranes with precisely designed compositions and structures for achieving desired filtration and separation performances. $^{4-9}$ Among them, graphene oxide (GO), prepared by the oxidation and exfoliation of graphite, is an excellent graphene derivative with great promise as a two-dimensional (2D) building block for the construction of separation membranes toward practical applications owing to its characteristics, such as ease of large-scale synthesis and functionalization. ${ }^{10-13}$ Because of the liquid-phaseprocessing feasibility, large-area and mechanically robust GO laminates with controllable thicknesses can be readily prepared via a series of liquid-phase membrane assembly techniques, such as drop-casting, ${ }^{14,15}$ vacuum filtration ${ }^{16,17}$ and spin-coating. ${ }^{18,19}$ In view of the structure of GO, various oxygen-containing functional groups (for example, hydroxyl, epoxy, carbonyl and carboxyl) decorate the graphene basal plane and its edges, resulting in numerous $s p^{2}$ aromatic clusters isolated within the $s p^{3} \mathrm{C}-\mathrm{O}$ matrix. ${ }^{20,21}$ On the basis of this unique structure, nanochannels for selective mass transport can be readily constructed by taking full advantage of the interlayer galleries between adjacent GO nanosheets (NS) within the lamellar membranes. The oxygen functionalities act as pillars to keep a relatively large interlayer spacing while the $s p^{2}$ clusters are connected across the stacking layers to form a nanocapillary network through

\footnotetext{
${ }^{1}$ State Key Laboratory of New Ceramics and Fine Processing, School of Materials Science and Engineering, Tsinghua University, Beijing, China; ${ }^{2}$ International Center for Materials Nanoarchitectonics (WPI-MANA), National Institute for Materials Science (NIMS), Ibaraki, Japan and ${ }^{3}$ Center for Nano and Micro Mechanics, Tsinghua University, Beijing, China Correspondence: Dr R Ma, International Center for Materials Nanoarchitectonics (WPI-MANA), National Institute for Materials Science (NIMS), 1-1 Namiki, Tsukuba, Ibaraki 305-0044, Japan.

E-mail: MA.Renzhi@nims.go.jp

or Professor H Zhu, State Key Laboratory of New Ceramics and Fine Processing, School of Materials Science and Engineering, Tsinghua University, Beijing 100084, China. E-mail: hongweizhu@tsinghua.edu.cn

Received 5 November 2015; revised 21 January 2016; accepted 31 January 2016
} 
which water molecules can be transported unimpededly, ${ }^{22}$ but solutes larger than $0.9 \mathrm{~nm}$ in diameter are blocked completely, ${ }^{23}$ showing potential in filtration and separation applications.

Typically, there are three principles for the design of separation membranes: steric hindrance, electrostatic and chemical interactions. Owing to the unique structures of GO-NS and the constructed lamellar membranes, the above three principles can be integrated into one piece of GO membrane, endowing great potential for multifunctional separation. For steric hindrance, all the species in solution with dimensions of $>0.9 \mathrm{~nm}$ can be sieved out by the nanocapillaries within the GO membranes. ${ }^{23}$ However, the critical size of $0.9 \mathrm{~nm}$ is too large for the separation of small hydrated ions. Therefore, GO membranes have been used mainly for blocking large organic dyes and nanoparticles thus far, ${ }^{24-26}$ whereas for most commonly available ions in solutions, GO membranes fail to act as a molecular sieve. Considering the charged nature of ions, it is possible to utilize electrostatic interactions between ionized oxygen functional groups (for example, carboxyl) and ions to generate selectivity for the filtration and separation of ions with different valences. However, according to Coulomb's law of discrete charge systems, the electrostatic force confronted by monovalent, divalent and trivalent ions in a fixed charge system (for example, a negatively charged GO membrane in water) lies in the order of 1:2:3. If the physical size effect of the nanocapillaries, chemical interactions (for example, hydrogen bonding, van der Waals interaction, cation- $\pi$ interaction and coordination), electrostatic screening (from ion hydration) and electrostatic resistance (from counter-ions) are not considered, the ion selectivity of GO membranes originating from electrostatic forces will lie in the order of 6:3:2 (the inverse form of electrostatic forces). This is too small to afford a significant separation of ions bearing various valences during the transport processes. Therefore, the development of GO-based separation membranes that function according to the charge of ions is limited by the relatively small differences in selectivity.

With respect to chemical interactions, previous studies have demonstrated that GO membranes can afford excellent selectivity toward various ions based on the coordination interactions of transition metal cations with oxygen-containing functional groups and the cation- $\pi$ interactions of alkali and alkaline earth cations with $s p^{2}$ aromatic clusters. ${ }^{27-29}$ On one hand, the chemical interactions between ions and GO membranes are complex and the balance between diverse chemical interactions and ion hydration-dehydration effect in nanochannels should be taken into full consideration, which requires substantial fundamental research before the selection of GO membranes for specific separation tasks. On the other hand, the relative selectivity among different ions is still below expectation, which awaits further enhancement for highly efficient separation goals. Therefore, GO membranes are highly suitable for blocking large organic molecules and nanoparticles based on steric hindrance, while the development of GO-based membranes with the ability to effectively separate various small ions according to a simple criterion (for example, only the charge of ions) is crucial for engineeringrelevant filtration and separation applications.

The interlayer galleries in GO laminates offer an advantageous platform for hetero-intercalation of guest species to improve or extend the functions of GO membranes. For example, by intercalating positively charged copper hydroxide nanostrands into GO laminates, followed by partially reducing and then dissolving the copper hydroxide nanostrands, the resulting nanostrand-channeled GO membranes offer a 10-fold enhancement in permeance without sacrificing the rejection rate of organic molecules compared with pristine GO membranes. ${ }^{30}$ By intercalating carbon nanotubes into GO layers, the water permeance can be enhanced significantly compared with pure GO membranes, whereas the rejection rates toward large organic molecules are still high. ${ }^{31,32}$ Recently, we intercalated monolayer titania-NS into GO laminates, followed by UV-induced photocatalytic reduction, and found that as-prepared reduced $\mathrm{GO} /$ titania hybrid membranes showed excellent ion rejection performance while the high water permeance of GO membranes could be preserved. ${ }^{33}$ These studies all indicate that by intercalating other functional nanomaterials into GO laminates, excellent solution-based separation performances can be achieved. However, in terms of separating small target ions from mixtures according to their charges, the above strategies are not effective. Functional nanomaterials with the ability to introduce strong electrostatic interactions and interfacial modifications that may further give rise to significant selectivity in permeation rates should be explored as a novel guest intercalant to form hybrids with GO-NS.

Layered double hydroxides (LDH), ${ }^{34-38}$ expressed by a formula of $\left[\mathrm{M}^{2+}{ }_{1-x} \mathrm{M}^{3+}{ }_{x}(\mathrm{OH})_{2}\right]^{x+}\left[\mathrm{A}^{n-}{ }_{x / n}\right]^{x-} \cdot m \mathrm{H}_{2} \mathrm{O}$, are a class of layered compounds composed of positively charged host layers and charge-balancing interlayer anions. The LDH crystallites can be exfoliated into the ultimate 2D NS with unilamellar thickness and high positive charge density, which represent another new type of 2D nanomaterial. ${ }^{39-45}$ Because of the in-plane positive charges of LDH-NS, superlattice composites can be expected via alternative face-to-face assembly of cationic LDH-NS and anionic GO-NS on a molecular scale. ${ }^{46,47}$ With these superlattice units, a macroscopic membrane may be constructed, in which the cationic LDH-NS are intercalated uniformly into the GO galleries, acting as both strengthening phases for electrostatic interactions and interlayer phases for modifying the physicochemical properties of the nanochannels. This unique structural feature may give rise to significant separations of small ions solely dependent on their charges.

In this work, large-area freestanding hybrid membranes consisting of anionic GO and cationic $\mathrm{Co}-\mathrm{Al}$ (or $\mathrm{Mg}-\mathrm{Al}$ ) LDH-NS superlattice units were prepared for the first time, which involved several key steps, such as the formation of superlattice composites, the transformation from superlattice precipitates to dispersions and vacuum-assisted membrane assembly. The concentration gradient-driven ion diffusion through the NS-heteroassembled hybrid membranes was investigated, as illustrated in Figure 1a, which shows that the cations bearing various valences can be effectively separated strictly according to their charges, independent of the cationic and charge-balancing anionic species. The relative selectivity between monovalent and trivalent cations for GO/LDH-NS hybrid membranes containing either Co-Al or $\mathrm{Mg}-\mathrm{Al}$ components is as high as 30 , which is not achievable by GO multilayer stacks, LDH-NS multilayer stacks or their bulk-stratified membranes. This indicates that the synergistic effect originating from the molecular-level heteroassembly of GO and LDH-NS has a dominant role in the high-performance charge-guided ion filtration and separation processes. These excellent properties make GO/LDH-NS hybrid membranes promising in applications such as wastewater treatment and reuse, chemical refinement and biomimetic selective ion transport for biological uses.

\section{MATERIALS AND METHODS}

\section{GO-NS preparation}

Graphite oxide was prepared by the modified Hummers' method using natural graphite as the source, which was subsequently exposed to potassium permanganate, sodium nitrite and concentrated sulfuric acid for oxidation. ${ }^{48}$ The obtained brown-colored graphite oxide flakes were exfoliated in water by sonication to yield monolayer GO-NS. ${ }^{49}$ 
a

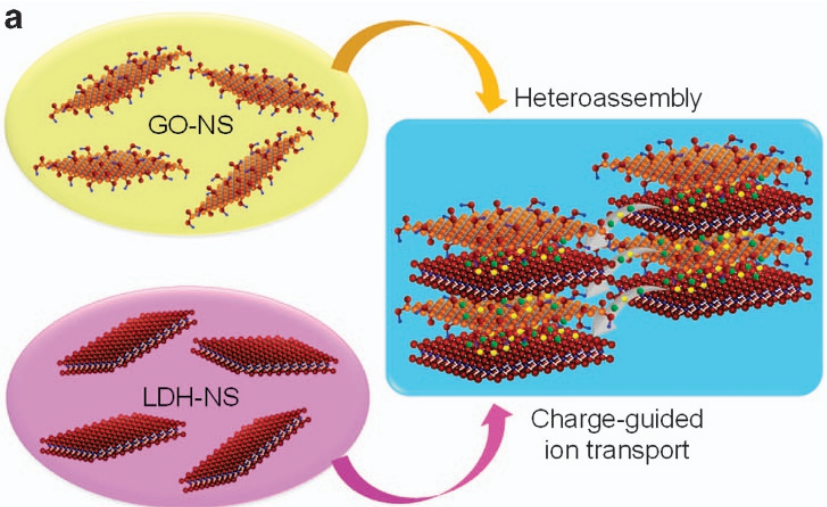

b

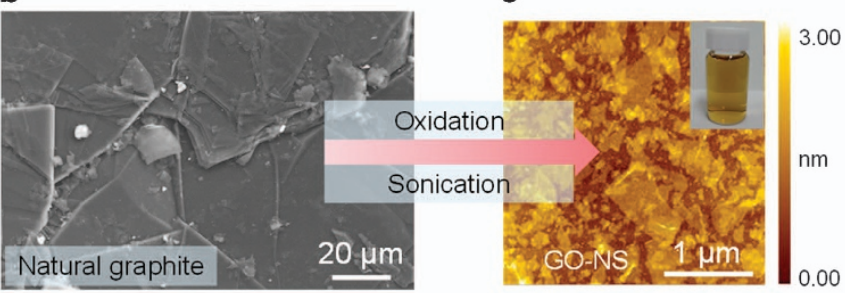

d

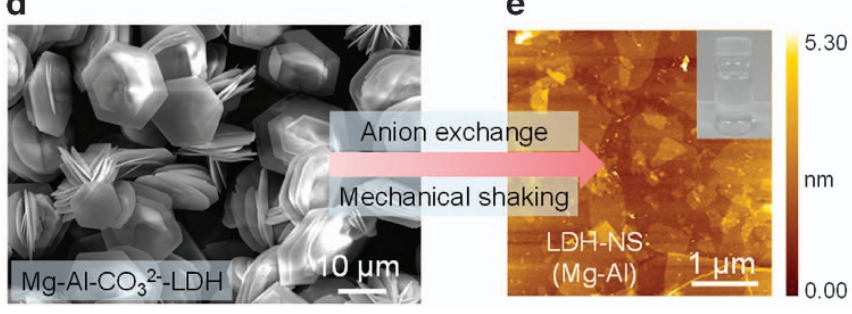

f

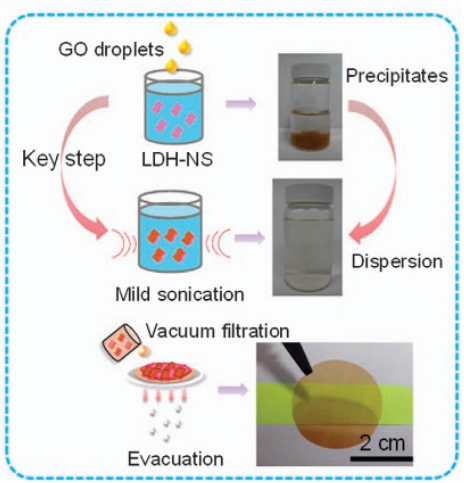

g

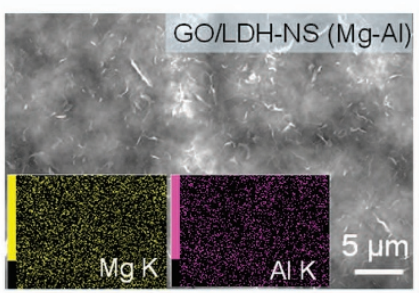

h

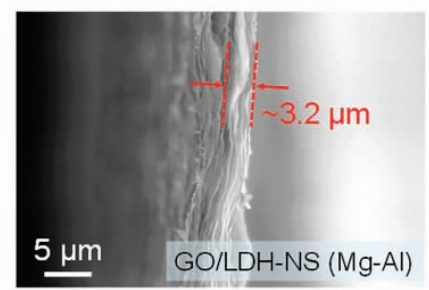

Figure 1 (a) Schematic diagrams illustrating the heteroassembly of GO and LDH-NS into molecular-scale superlattice units and the charge-guided ion filtration and separation process. (b) SEM image of the natural graphite flakes and (c) atomic force microscopy (AFM) image of the exfoliated GO-NS. (d) SEM image of the well-crystallized LDH (Mg-Al) hexagon platelets and (e) AFM image of the exfoliated Mg-Al LDH-NS. The insets in (c) and (e) are photographs of GO and Mg-Al LDH-NS suspensions. (f) Synthesis procedure for the GO/LDH-NS hybrid membrane and corresponding photographs showing the obtained precipitates, re-dispersed suspension and the final freestanding membrane after each step. ( $\mathbf{g}$ and $\mathbf{h}$ ) SEM characterizations of the morphology and cross-section of the as-prepared GO/LDH-NS hybrid membrane (Mg-Al). The insets in (g) show energy-dispersive spectroscopy mappings of Mg and Al elements.

\section{Co-Al and Mg-Al LDH-NS preparation}

The preparation of $\mathrm{Co}-\mathrm{Al}$ and $\mathrm{Mg}-\mathrm{Al} \mathrm{LDH}-\mathrm{NS}$, which followed previously developed soft-chemical procedures, ${ }^{40,41}$ involved the synthesis of $\mathrm{Co}-\mathrm{Al}-\mathrm{CO}_{3}{ }^{2-}$ and $\mathrm{Mg}-\mathrm{Al}-\mathrm{CO}_{3}{ }^{2-} \mathrm{LDH}$ hexagon platelets by refluxing and hydrothermal treatment, respectively, decarbonation and anion exchange into $\mathrm{NO}_{3}{ }^{-}$forms by a salt-acid treatment with $\mathrm{NaNO}_{3}$ and $\mathrm{HNO}_{3}$ mixed solutions under mechanical shaking, and delamination/exfoliation of Co-Al- $\mathrm{NO}_{3}{ }^{-}$and $\mathrm{Mg}-\mathrm{Al}-\mathrm{NO}_{3}{ }^{-} \mathrm{LDH}$ platelets in formamide under mechanical shaking.

\section{GO/LDH-NS hybrid membrane preparation}

First, electrostatic heteroassembly of anionic GO and cationic LDH-NS into superlattice units was performed by dropwise addition of a $0.1 \mathrm{mg} \mathrm{ml}^{-1} \mathrm{GO}$ aqueous suspension $\left(25 \mathrm{ml}\right.$ in total) into $7.5 \mathrm{ml}$ of a $1 \mathrm{mg} \mathrm{ml}^{-1} \mathrm{LDH}-\mathrm{NS}$ formamide suspension under vigorous stirring. The mass ratio of GO and LDH-NS (1:3) was determined by a hypothesized area matching model (see the Supplementary Table 1). The collected precipitates were centrifuged and washed with water and ethanol several times to remove excess ions and NS. Next, the precipitates were mildly sonicated in water for $\sim 10 \mathrm{~min}$ to homogeneously disperse the superlattice units. The obtained suspension was immediately filtered through a microfilter with a thickness of $\sim 100 \mu \mathrm{m}$ and a pore size of $\sim 0.2 \mu \mathrm{m}$ (porosity: $\sim 80 \%$ ) under vacuum to form a GO/LDH-NS hybrid membrane on the microfilter. After drying in air at $55^{\circ} \mathrm{C}$ for $1 \mathrm{~h}$, the hybrid membrane could be easily detached off from the microfilter, and a piece of freestanding membrane was obtained. For comparison, GO multilayer stacks and LDH-NS multilayer stacks were prepared by vacuum-assisted filtering $25 \mathrm{ml}$ of the $0.1 \mathrm{mg} \mathrm{ml}^{-1} \mathrm{GO}$ aqueous suspension and $7.5 \mathrm{ml}^{\text {of the }} 1 \mathrm{mg} \mathrm{ml}^{-1}$ LDH-NS (for example, Co-Al) formamide suspension through a microfilter, respectively. The bulk-stratified membranes consisting of GO multilayer stacks and LDH-NS multilayer stacks were prepared using a two-step vacuumfiltration method: first, $7.5 \mathrm{ml}$ of the $1 \mathrm{mg} \mathrm{ml}^{-1}$ LDH-NS formamide suspension was vacuum-filtered through a microfilter; after drying, $25 \mathrm{ml}$ of the $0.1 \mathrm{mg} \mathrm{ml}^{-1} \mathrm{GO}$ aqueous suspension was filtered through the same microfilter. It should be noted that all the as-prepared NS-based lamellar membranes can be stable in water for a long time, as demonstrated by the linear relationship between salt permeance and time for several tens of hours and the absence of obvious cracks or tears visible to the naked eye after several weeks.

\section{Concentration gradient-driven ion diffusion tests}

The ion transmembrane diffusion experiments were conducted with a self-made permeation apparatus, which has also been used in our previous studies. ${ }^{27-29}$ Briefly, the source and drain reservoirs were separated by a plastic plate with a leak hole $(\sim 5 \mathrm{~mm}$ in diameter) in the center. A piece of membrane was sealed with double-sided copper tape that was drilled with the same sized hole $(\sim 5 \mathrm{~mm}$ in diameter) so that the membrane could directly connect the source and drain solutions and facilitate ion transmembrane permeation 
through an effective leak area of $\sim 19.6 \mathrm{~mm}^{2}$. During the runs, $120 \mathrm{ml}$ of a $0.1 \mathrm{M}$ certain source solution and deionized water were injected at the same speed into the source and drain reservoirs, respectively, and the conductivity variations of the drain solution were measured by a conductivity meter with time, based on which the salt transmembrane permeances as a function of time were calculated and plotted.

\section{RESULTS AND DISCUSSION}

\section{Preparation of GO-NS, LDH-NS and GO/LDH-NS hybrid} membranes

Figures $1 \mathrm{~b}$ and $\mathrm{c}$ show the scanning electronic microscopy (SEM) and atomic force microscopy images of the graphitic precursors and the exfoliated GO-NS. The graphite precursors possess a platelet-shaped morphology and a lamellar structure (Figure 1b). After oxidation by potassium permanganate, sodium nitrite and concentrated sulfuric acid, followed by exfoliation in water, monolayer GO-NS with a typical lateral size of several microns to several hundred nanometers and a thickness of $\sim 1 \mathrm{~nm}$ are generally obtained (Figure 1c).

Figures $1 \mathrm{~d}$ and e show the SEM and atomic force microscopy images of the $\mathrm{Mg}-\mathrm{Al}-\mathrm{CO}_{3}{ }^{2-} \mathrm{LDH}$ precursors and the exfoliated NS (characterizations for the corresponding $\mathrm{Co}-\mathrm{Al}$ forms are shown in Supplementary Figure S1). The LDH platelets are well crystallized, with a regular hexagonal shape and lateral dimensions on the order of several microns (Figure 1d and Supplementary Figure S1a). After decarbonation and anion exchange into the $\mathrm{NO}_{3}{ }^{-}$forms, followed by exfoliation in formamide, monolayer LDH-NS structures with typical lateral dimensions of several microns and a thickness of $\sim 0.8 \mathrm{~nm}$ are obtained (Figure 1e and Supplementary Figure S1b). The thicknesses of both GO and LDH-NS as measured by atomic force microscopy are slightly greater than their crystallographic thicknesses, a possible effect of surface adsorption. It should be noted that the thorough decarbonation and exchange into the $\mathrm{NO}_{3}{ }^{-}$form is the prerequisite for efficient exfoliation of LDH platelets into monolayer NS because of the high affinity and strong coupling of $\mathrm{CO}_{3}{ }^{2-}$ ions in between LDH host layers, which impede the delamination of the $\mathrm{LDH}$ platelets. As shown in the X-ray diffraction (XRD) patterns in Supplementary Figures S2a and $c$, after mechanical shaking in $\mathrm{NaNO}_{3} / \mathrm{HNO}_{3}$ mixtures, both the $\mathrm{Mg}-\mathrm{Al}-\mathrm{CO}_{3}{ }^{2-}$ and $\mathrm{Co}-\mathrm{Al}-\mathrm{CO}_{3}{ }^{2-} \mathrm{LDH}$ platelets are completely exchanged into their $\mathrm{NO}_{3}{ }^{-}$forms with an increased interlayer spacing of $\sim 0.87 \mathrm{~nm}$, compared with $\sim 0.73 \mathrm{~nm}$ in the $\mathrm{CO}_{3}{ }^{2-}$ forms. $\mathrm{No} \mathrm{CO}_{3}{ }^{2-}$ - $\mathrm{LDH}$ signal is observed at $\sim 12^{\circ}$. Notably, as shown in Supplementary Figures S2b and d, after decarbonation and anion exchange, the morphology and dimensions of Co-Al LDHs are nearly unchanged, whereas some of the $\mathrm{Mg}-\mathrm{Al} \mathrm{LDHs}$ are torn into irregular or round shapes because of their larger precursor sizes.

With these two types of oppositely charged 2D building blocks, heterostructured flocculates were synthesized by the dropwise addition of GO aqueous suspension into an LDH-NS formamide suspension under vigorous stirring (1:3 in mass ratio according to a hypothesized area matching model, see Supplementary Table 1), followed by repeated centrifugation and washing with water and ethanol to remove excess NS and ions. To further construct a high-quality membrane for possible filtration and separation applications with these flocculates, a key step in the fabrication procedure is to re-disperse the collected precipitates in water via mild sonication, followed by vacuum filtering through a microfilter. The sonication time is crucial because the precipitates cannot be dispersed homogeneously if the duration of sonication is too short, but the pristine heterostructure may be destroyed if it is too long; either condition will seriously affect the quality of the as-prepared hybrid membranes. In this case, the sonication time was chosen to be $\sim 10 \mathrm{~min}$ for the best dispersion of the heterostructured precipitates without substantial damage. In addition, vacuum filtration should be performed immediately after the re-dispersing process because the precipitates will reemerge within a short time $(<30 \mathrm{~min})$. The detailed synthesis procedure is depicted in Figure 1f. After drying in air at $55^{\circ} \mathrm{C}$ for $1 \mathrm{~h}$, the as-prepared GO/LDH-NS hybrid membrane can be readily detached from the microfilter and is semitransparent, flexible, freestanding and mechanically robust. The area of the hybrid membrane depends on that of the microfilter, and the thickness depends on the amount of dispersion filtered. Typically, it takes several hours to prepare a micrometer-thick GO membrane via vacuum filtration. By contrast, the formation of a GO/LDH-NS hybrid membrane containing the same amount of GO-NS takes only 2-3 min, greatly raising the efficiency of membrane preparation. Therefore, this simple vacuum-assisted method for GO/LDH-NS hybrid membrane preparation is highly suitable for industrial-scale applications. Figures $1 \mathrm{~g}$ and $\mathrm{h}$ show the SEM characterizations for the morphology and cross-section of a GO/LDH-NS hybrid membrane (Mg-Al), revealing that the as-prepared hybrid membrane is continuous and uniform, with small wrinkles on the surface and a lamellar structure $\sim 3-\mu \mathrm{m}$ thick. The insets in Figure 1g show the energy-dispersive spectroscopy mappings of $\mathrm{Mg}$ and $\mathrm{Al}$ for the whole area, revealing that the LDH-NS are distributed uniformly within the entire membrane. Similar SEM characterizations and energy-dispersive spectroscopy mappings for the GO/LDH-NS hybrid membrane (Co-Al) are shown in Supplementary Figure S3.

\section{XRD characterizations for the structures of GO/LDH-NS hybrids} Structure evolution from GO/LDH-NS precipitates to hybrid membrane. The structure evolution from GO/LDH-NS heterostructured precipitates to a large-area hybrid membrane was studied by XRD (taking GO/Co-Al LDH-NS as an example), as shown in Figure 2a. After heteroassembly, followed by repeated washing and centrifugation, the precipitates collected by directly pouring out the supernatant show a superlattice structure with an interlayer spacing of $\sim 1.1 \mathrm{~nm}$ (curve 1 in Figure 2a). If the crystallographic thicknesses of GO $(0.78 \mathrm{~nm}$, shown in Figure $2 \mathrm{~d})$ and the $\mathrm{LDH}$ host layer $(0.48 \mathrm{~nm})$ are taken into account, a basal spacing of $1.26 \mathrm{~nm}$ is expected for a superlattice structure containing intercalated water, which is close to the value obtained here. The slightly reduced basal spacing can be attributed to the interlayer contraction originating from electrostatic attractions between the oppositely charged $\mathrm{NS}^{46,47}$ and the loss of intercalated water in the interlayer galleries. The latter can be demonstrated by the relatively hydrophobic surface of the GO/LDH-NS hybrid membrane (Co-Al) (contact angle: $\sim 64^{\circ}$ ) compared with that of the GO membrane (contact angle: $\sim 44^{\circ}$ ). In fact, for an anhydrous GO membrane, the interlayer spacing was reported to be $\sim 0.6 \mathrm{~nm},{ }^{50}$ giving rise to a basal spacing of $1.08 \mathrm{~nm}(0.6 \mathrm{~nm}+0.48 \mathrm{~nm})$ for an ideal superlattice structure of GO/LDH-NS without any intercalants, which conforms exactly with the case here $(\sim 1.1 \mathrm{~nm})$, indicating that few water molecules are intercalated between GO and LDH-NS in the superlattice composites.

Interestingly, for the precipitates collected by removing the supernatant via vacuum filtering through a microfilter, the peak assigned to the interlayer spacing of a superlattice structure disappears. Instead, a new peak located at $\sim 11.4^{\circ}$ emerges (curve 2 in Figure $2 \mathrm{a}$ ). Changing the method of removing supernatant cannot destroy the superlattice structure, but the significant differences in XRD patterns indicate that the stacking manner of the superlattice units has been altered greatly. By directly pouring out the supernatant, the superlattice units are not directionally stacked (panel 1 in Figure $2 b$ ), and the characteristics of 

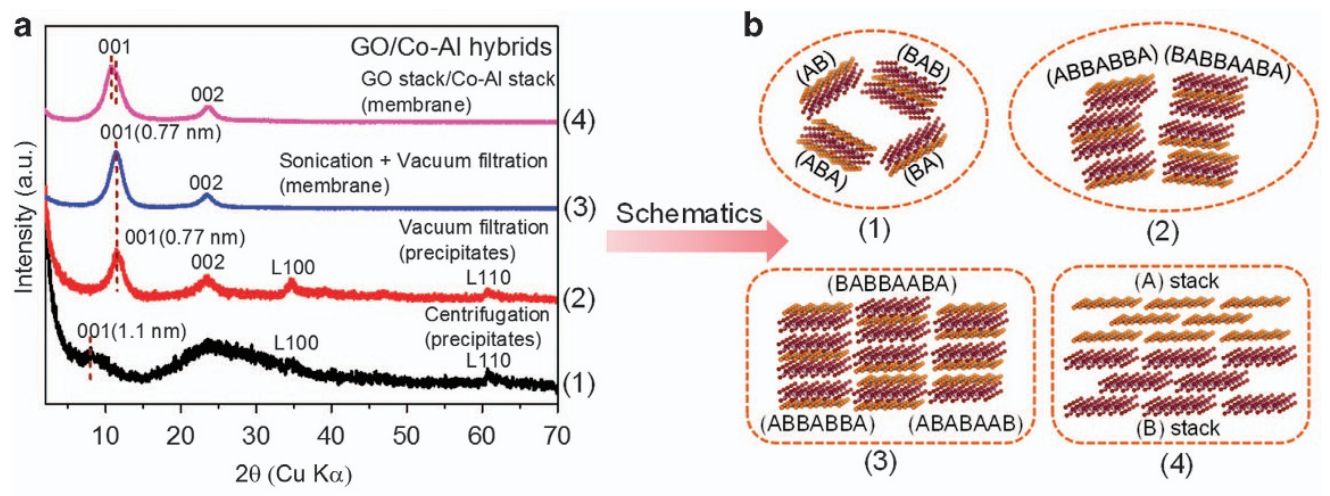

(3) Schematics

(1)

(2)

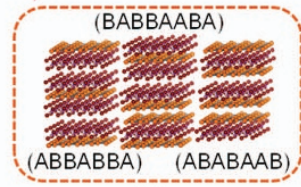

(3)

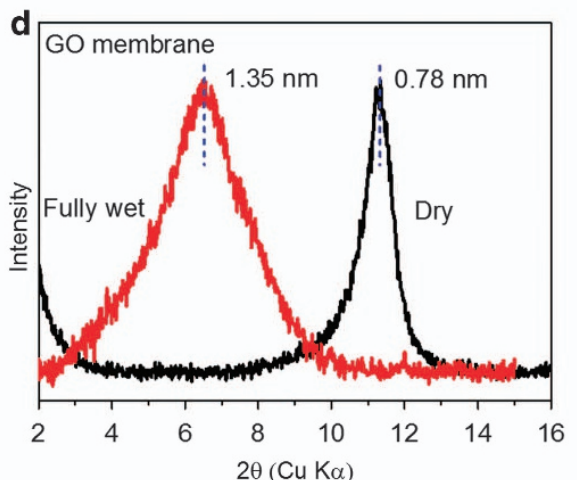

Figure 2 (a) XRD patterns of GO/Co-Al precipitates collected by centrifugation (curve 1) or vacuum-filtration (curve 2), GO/Co-Al hybrid membrane (curve 3) and the bulk-stratified membrane consisting of a GO multilayer stack (top) and a Co-Al multilayer stack (bottom) (curve 4). (b) Schematics illustrating the possible structures of the four cases in (a). XRD patterns of (c) the GO/LDH-NS hybrid membrane (Co-Al) and (d) the pure GO membrane in dry (humidity: $\sim 40 \%)$ and fully wet states, respectively.

the superlattice structure can be generally revealed in the XRD pattern (curve 1 in Figure 2a). On the other hand, by collecting the precipitates via vacuum filtration, the evacuation force would drive the directional stacking of superlattice units with various stacking sequences and compositions (panel 2 in Figure 2b), resulting in a long-range disordered stacking structure and the disappearance of the peak assigned to the superlattice structure in the XRD pattern (curve 2 in Figure 2a). In this case, the interlayer spacing of $\sim 0.77 \mathrm{~nm}$ (curve 2 in Figure 2a) represents the average interlayer distance between adjacent $2 \mathrm{D}$ building blocks (for example, GO-LDH, GO-GO and $\mathrm{LDH}-\mathrm{LDH}$ with the intercalated $\mathrm{NO}_{3}{ }^{-}$or $\mathrm{CO}_{3}{ }^{2-}$ counter-ions).

After mild sonication, followed by vacuum filtration to form largearea GO/LDH-NS hybrid membranes, the peaks in the XRD pattern (curve 3 in Figure 2a) are not changed compared with those in curve 2 in Figure 2a, except for the disappearance of the in-plane reflections, L100 and L110, from LDH-NS. This indicates that, compared with collecting precipitates via vacuum filtration, the mild sonication followed by vacuum-filtration processes for membrane preparation lead to more ordered stacking of superlattice units (panel 3 in Figure $2 \mathrm{~b}$ ), characterized with the disappearance of in-plane reflections from LDH-NS in the XRD pattern. It should be noted that the superlattice units in the pristine precipitates are not damaged upon sonication, which can be demonstrated by the reemergence of precipitates within a short time $(<30 \mathrm{~min})$ in the re-dispersed suspension with a nearly unchanged XRD pattern, as shown in Supplementary Figure S4. This indicates that the as-prepared GO/LDH-NS hybrid membranes are indeed constructed by anionic GO and cationic LDH-NS superlattice units. For control, a bulk-stratified membrane consisting of a GO multilayer stack (top) and an LDH-NS multilayer stack (bottom) was prepared and subjected to XRD characterization (curve 4 in Figure 2a). This revealed that the 001 peak is broadened and asymmetric, with two components assigned to the interlayer distances of the Co-Al LDH-NS multilayer stack $\left(\sim 0.82 \mathrm{~nm}\right.$, possibly intercalated with $\mathrm{NO}_{3}{ }^{-}$and $\mathrm{CO}_{3}{ }^{2-}$ counter-ions) and the GO multilayer stack $(\sim 0.78 \mathrm{~nm})$, respectively (see panel 4 in Figure 2b), which is different from the cases of the GO/Co-Al LDH-NS hybrid precipitates and membranes.

Structural response of the GO/LDH-NS hybrid membrane to humidity variations compared with pure GO membrane. Next, the structural response of GO/LDH-NS hybrid membrane to humidity variations was characterized by XRD, as shown in Figure 2c. Notably, the interlayer spacing of the hybrid membrane is insensitive to humidity variations. By immersing the hybrid membrane in water for a fully wet state, the interlayer spacing is not changed compared with that of the dry state, which is consistent with the contact angle measurements showing that the hybrid membrane is relatively hydrophobic. This property is crucial for solution-based mass transport applications because the insensitivity of the interlayer spacing to humidity variations ensures the structural stability for long-term and efficient operations in water. By contrast, after immersing the pure GO membrane in water for a fully wet state, the interlayer spacing increases to $1.35 \mathrm{~nm}$ from $0.78 \mathrm{~nm}$ in the dry state, as shown in Figure $2 \mathrm{~d}$, demonstrating its structural instability in water. In addition, the large nanochannels $(0.95 \mathrm{~nm}=1.35-0.4 \mathrm{~nm}, 0.4 \mathrm{~nm}$ is the interlayer spacing of fully reduced GO membranes ${ }^{22,23}$ ) are suitable only for blocking large organic molecules and nanoparticles based on steric hindrance. 

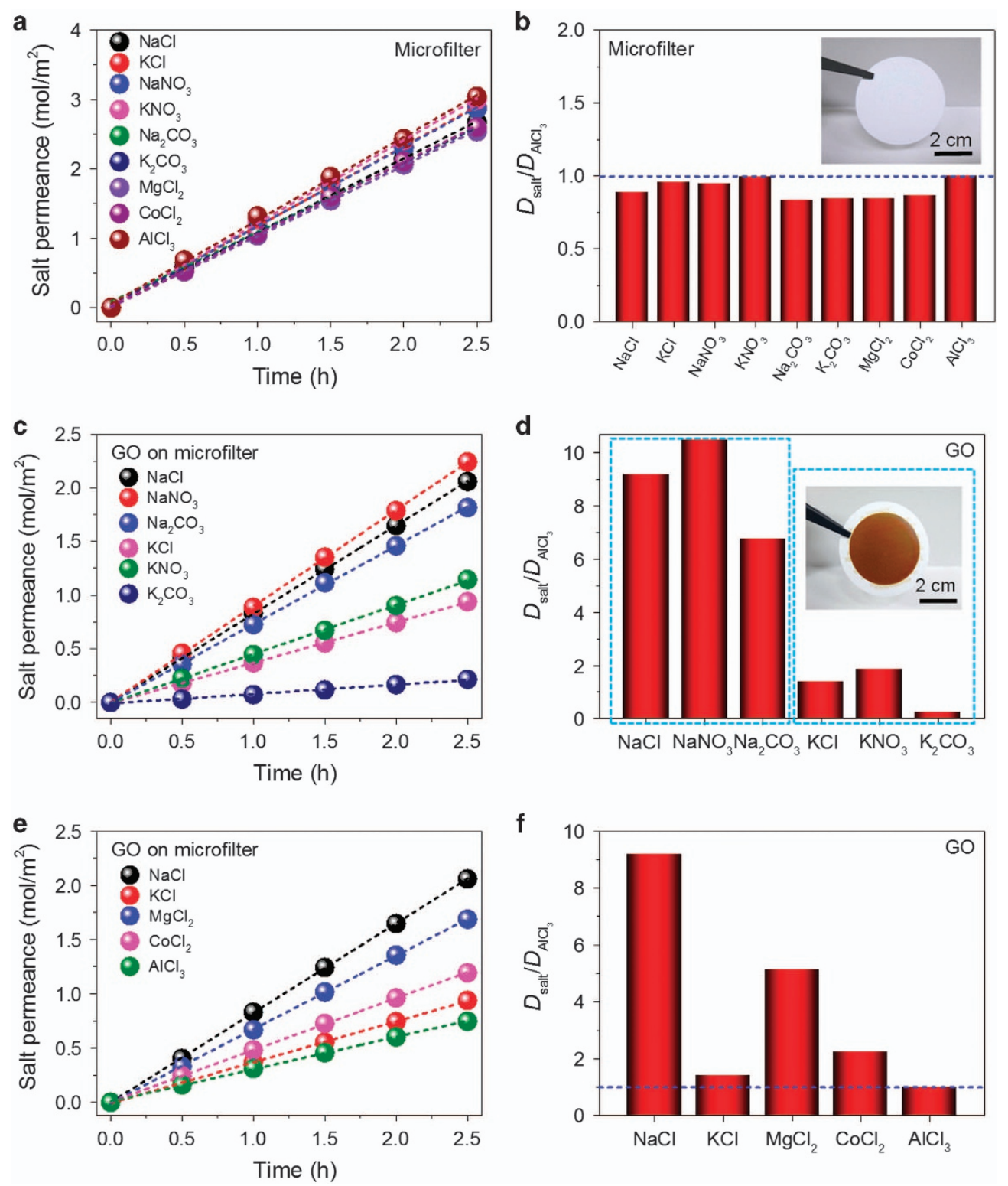

Figure 3 (a and $\mathbf{b}$ ) Salt permeations through bare microfilters and the corresponding diffusion coefficients (normalized by $\mathrm{AlCl}_{3}$ ). (c-f) Salt permeations through GO membranes on microfilters and the corresponding diffusion coefficients through GO membranes (normalized by $\mathrm{AICl}_{3}$ ). The insets in (b) and (d) are photographs of a bare microfilter and the GO membrane on a microfilter, respectively. The dashed boxes in (d) indicate the diffusion coefficients of sodium salts and potassium salts with various anions, respectively.

At this point, we have demonstrated that GO/LDH-NS hybrid membranes possess several structural advantages for possible filtration and separation applications: (i) the intercalation of cationic LDH-NS into GO layers by forming local superlattice units introduces high positive charge densities, which may facilitate the effective separation of ions based on electrostatic interactions; (ii) the relatively hydrophobic interfaces of the hybrid membranes may facilitate the dehydration of ions, further weakening the electrostatic screening induced by hydration shells and enhancing the diverse interactions of ions with the membranes for more effective separation; (iii) the narrowing of the nanochannels (the average interlayer spacing: $\sim 0.77 \mathrm{~nm})$ compared with pure GO membranes $(\sim 1.35 \mathrm{~nm}$ in fully wet state) may enable the effective separation of small ions based on steric hindrance; (iv) the insensitivity of the interlayer spacing to humidity variations ensures the structural stability in water, which is the foundation for practical applications. In the following section, the concentration gradient-driven ion diffusions through the GO/LDH-NS hybrid membranes were systematically investigated in comparison with bare microfilters, GO multilayer stacks, LDH-NS multilayer stacks and their bulk-stratified membranes.

\section{Concentration gradient-driven ion transmembrane diffusions}

Experimental setup. The ion transmembrane permeation experiments were performed by sealing a piece of membrane to separate the source reservoir from the drain reservoir containing $120 \mathrm{ml}$ of a certain $0.1 \mathrm{M}$ salt solution and deionized water, respectively (see the Experimental Section). The ion transmembrane permeations were investigated by measuring the conductivity variations in drain solutions according to the fact that, in a dilute solution, the conductivity varies linearly with the salt concentration, as demonstrated in Supplementary Figure S5. 
On the basis of the conductivity measurements, salt permeances as a function of time can be calculated and plotted, and the transmembrane diffusion coefficients of various salts can be calculated according to Fick's first law: $J=-D \Delta c / l$, where $J$ is transmembrane flux, $D$ is diffusion coefficient, $c$ is concentration and $l$ is membrane thickness. In all the experiments, the calculated diffusion coefficients for various salts were normalized by that of $\mathrm{AlCl}_{3}$ for better comparison.

Ion permeations through bare microfilters. First, the salt permeations through bare microfilters were investigated as a control. The thickness of the microfilters used in all the experiments is $\sim 100 \mu \mathrm{m}$, as shown in Supplementary Figure S6a, based on which the diffusion coefficients of various salts through bare microfilters can be calculated. As shown in Figures $3 a$ and $b$, various salts permeate through bare microfilters with nearly the same permeation rates, indicating that the microfilters have no selectivity.
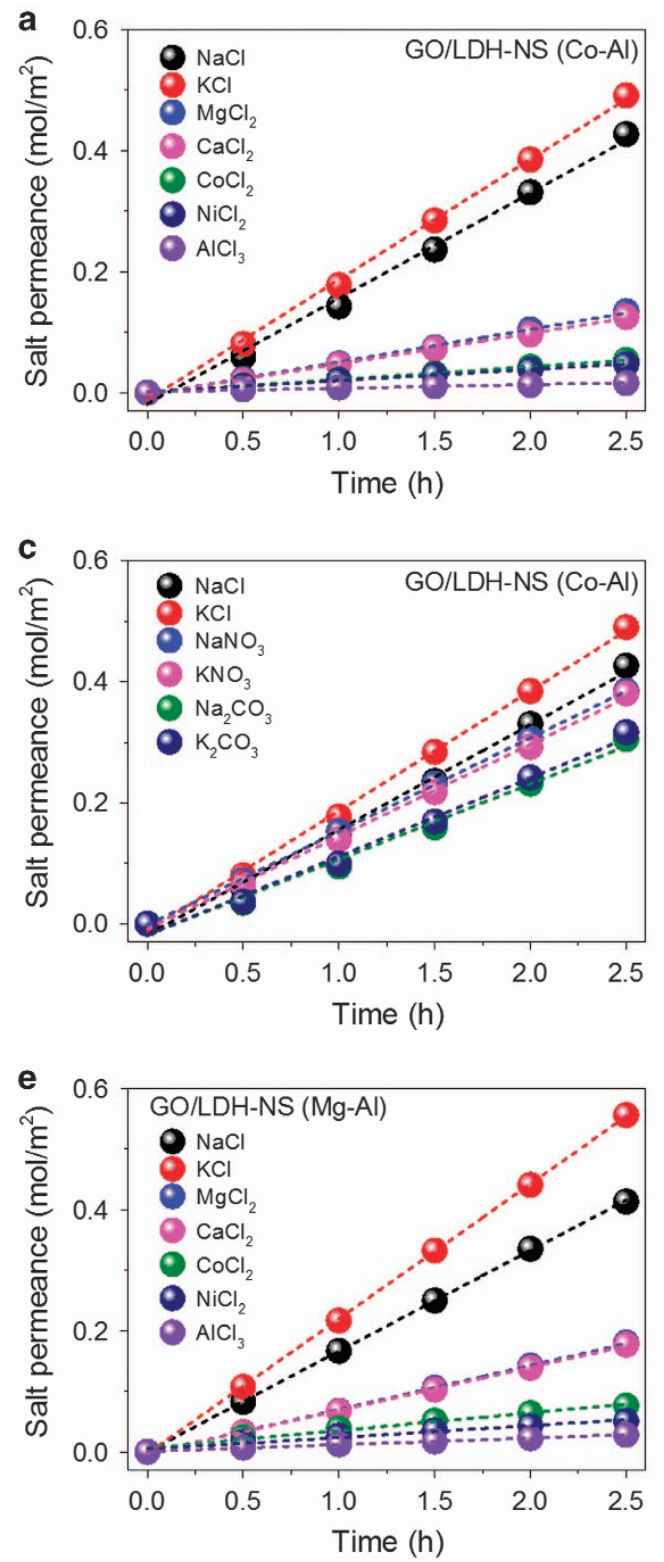

Ion permeations through GO membranes. Next, pure GO membranes were prepared on microfilters with a coverage density of $2.6 \mathrm{~g} \mathrm{~m}^{-2}$ via vacuum filtration. The SEM characterizations on the morphology and cross-section of a representative GO membrane are shown in Supplementary Figures S6b and c, revealing a wrinkled surface and a lamellar structure with a thickness of $\sim 1.6 \mu \mathrm{m}$. With these GO membranes on microfilters, various salts were allowed to permeate under the same concentration gradient $(0.1 \mathrm{M})$. The salt permeances are plotted as a function of time in Figures $3 c$ and $e$, and the corresponding diffusion coefficients through the GO membranes were calculated by extracting the effect of microfilters, as shown in Figures $3 \mathrm{~d}$ and $\mathrm{f}$ (detailed calculation procedures with schematics are in Supplementary Figure S7).

This reveals that excellent selectivity can be achieved with GO membranes toward the permeations of various salts based on diverse
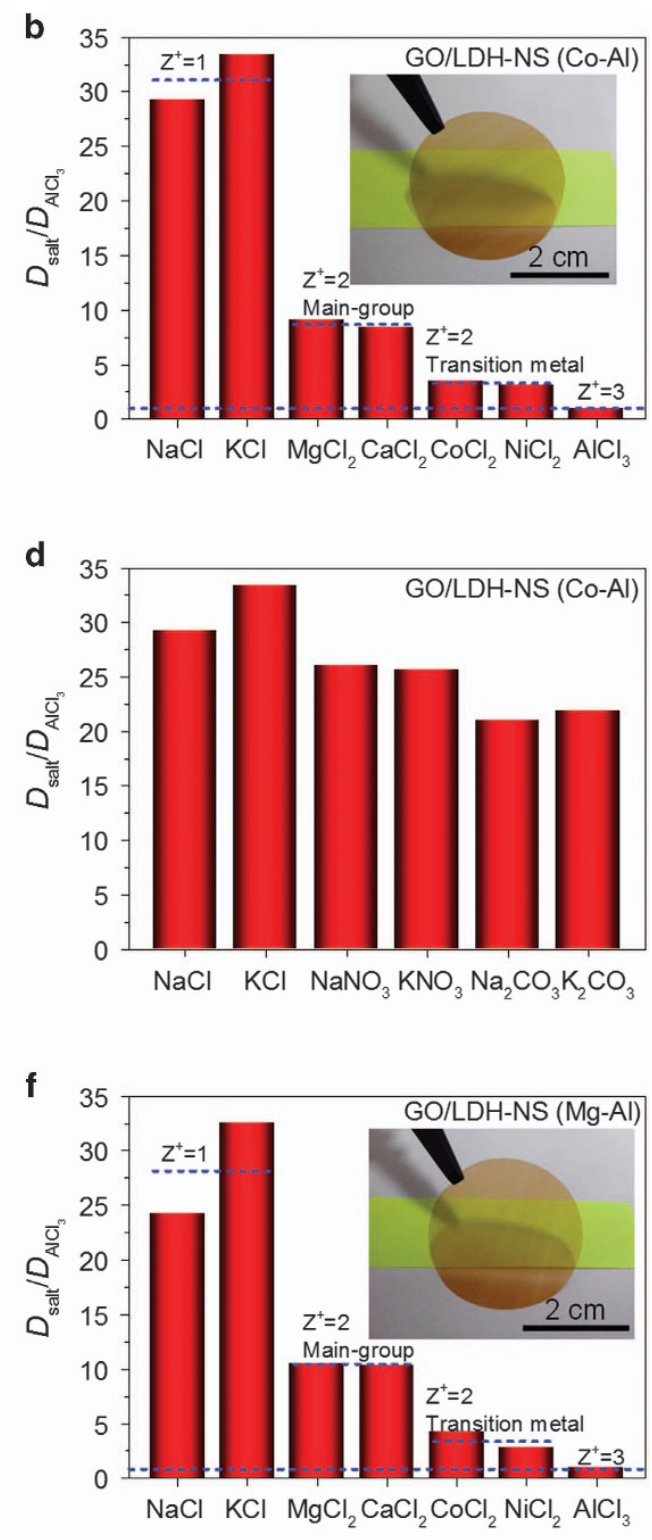

Figure 4 (a-d) Salt permeations through GO/LDH-NS hybrid membranes (Co-Al) and the corresponding diffusion coefficients (normalized by $\mathrm{AlCl}_{3}$ ). (e and f) Salt permeations through GO/LDH-NS hybrid membranes (Mg-Al) and the corresponding diffusion coefficients (normalized by $\mathrm{AlCl}_{3}$ ). The insets in (b) and (f) are photographs of GO/LDH-NS hybrid membranes containing Co-Al and Mg-Al LDH-NS, respectively. 
chemical and electrostatic interactions, beyond the functioning stage of steric hindrance effect of $s p^{2}$ nanocapillaries: ${ }^{23}$ (i) the electrostatic repulsions between anions and ionized carboxyl groups on GO (for example, electrostatic strengths: $\mathrm{CO}_{3}{ }^{2-}>\mathrm{Cl}^{-}>\mathrm{NO}_{3}{ }^{-}$) give rise to significant selectivity toward the permeations of salts with various anions (permeation rates: $\mathrm{CO}_{3}{ }^{2-}$ salts $<\mathrm{Cl}^{-}$salts $<\mathrm{NO}_{3}{ }^{-}$salts), as shown in Figures $3 \mathrm{c}$ and d; (ii) the diverse chemical interactions between cations and GO give rise to significant selectivity toward the permeations of various metal salts with the same anions, as shown in Figures $3 \mathrm{c}-\mathrm{f}$. In detail, because of the stronger cation- $\pi$ interaction of $\mathrm{K}^{+}$with the $s p^{2}$ nanocapillaries than that of $\mathrm{Na}^{+}$, which results in more effective anchoring of $\mathrm{K}^{+}$ions, the permeations of sodium salts are always faster than potassium salts, as shown in Figures $3 \mathrm{c}$ and d. For $\mathrm{Na}^{+}, \mathrm{Mg}^{2+}$ and $\mathrm{Al}^{3+}$ salts, the higher positive charges of main-group metal cations generally result in stronger cation- $\pi$ interactions, further leading to slower salt permeation rates $\left(\mathrm{Al}^{3+}\right.$ salts $<\mathrm{Mg}^{2+}$ salts $<\mathrm{Na}^{+}$ salts), as shown in Figures $3 e$ and $\mathrm{f}$. On the other hand, owing to the strong coordination interaction between transition metal cations $\left(\mathrm{Co}^{2+}\right)$ and the oxygen functional groups on GO, the permeation of $\mathrm{Co}^{2+}$ salts is slower than the same periodic and charged $\mathrm{Mg}^{2+}$ salts, as shown in Figures $3 \mathrm{e}$ and $\mathrm{f}$. These results are in good agreement with our previous studies. ${ }^{27-29}$

It should be noted that the diverse chemical and electrostatic interactions between ions and GO, which are mainly responsible for ion selectivity of GO membranes, are too complex for engineeringrelevant applications. The separation of various salts based on the permeation rate differences lacks a general criterion, which requires substantial fundamental work to be conducted before the use of GO membranes for a specific separation task. In addition, as shown in Figure $3 \mathrm{f}$, the relative selectivity of GO membranes toward various cations is not high enough, with the maximum relative selectivity of $\sim 9$ between $\mathrm{Na}^{+}$and $\mathrm{Al}^{3+}$, which should be enhanced further for highly efficient separation goals. Bearing the above limitations of GO membranes in mind, an improved GO-based hybrid membrane with the ability to more effectively separate small ions according to a simple criterion (for example, only the charge of ions) is highly desired.

Ion permeations through GO/LDH-NS hybrid membranes. As shown in Figures $4 \mathrm{a}$ and $\mathrm{b}$, the permeations of cations bearing various valences through GO/LDH-NS hybrid membranes (Co-Al) exhibit a strict charge-guided tendency, independent of the specific cation species, indicating that the cations can be effectively separated during the diffusion process only according to their charges, covering the shortcomings of pure GO membranes. The relative selectivity among monovalent, divalent and trivalent main-group metal cations lies in the order of $\sim 31: 8: 1$, beyond the predictions from Coulomb's law of discrete charge systems (maximum: 6:3:2), whereas the diffusions of transition metal cations are significantly slower than main-group metal cations with the same charges. These excellent results indicate that GO/LDH-NS hybrid membranes (Co-Al) are highly suitable for engineering-relevant filtration and separation applications, considering the high relative selectivity among cations with various valences and the even slower transport rates of transition metal cations. Notably, as shown in Figures $4 \mathrm{c}$ and $\mathrm{d}$, the permeations of cations (for example, $\mathrm{K}^{+}$and $\mathrm{Na}^{+}$) are nearly independent of the charge-balancing anion species (for example, $\mathrm{Cl}^{-}, \mathrm{NO}_{3}{ }^{-}$and $\mathrm{CO}_{3}{ }^{2-}$ ), indicating that ion permeation through the hybrid membrane is only positive chargedominated, which further simplifies the membrane selection criterion for specific ion separation tasks. Similar results have been obtained with GO/LDH-NS hybrid membranes (Mg-Al), as shown in Figures 4e and $\mathrm{f}$, indicating that the ion permeation properties of GO/LDH-NS hybrid membranes are general to this specific type of GO-based hybrid membrane, independent of the metal species in the host layers of LDH-NS.

Ion permeations through bare LDH-NS stacks and their bulk-stratified with GO stacks for control. Control experiments were conducted with bare Co-Al LDH-NS multilayer stacks and their bulk-stratified with GO multilayer stacks (mass ratio: 3:1, the same as GO/LDH-NS hybrid membranes), as shown in Supplementary Figure S8. The results indicate that the permeations of various salts through these two types of control membranes and the corresponding relative selectivity are insignificant compared with GO/LDH-NS hybrid membranes (Figure 4) and GO membranes (Figure 3). This can be attributed to the hydrophobic interlayer galleries within the LDH-NS multilayer stacks (contact angle: $\sim 82^{\circ}$ ), possibly originating from the formamide modification during the exfoliation process, that are difficult for all the salts to permeate. These results confirm that the LDH-NS multilayer stacks alone or their bulk-stratified with GO multilayer stacks cannot afford the same excellent ion filtration and separation performances as the GO/LDH-NS hybrid membranes, further indicating that the molecular-scale heteroassembly of GO and LDH-NS within the hybrid membrane synergistically dominates the high-performance chargeguided ion filtration and separation processes of GO/LDH-NS hybrid membranes. a

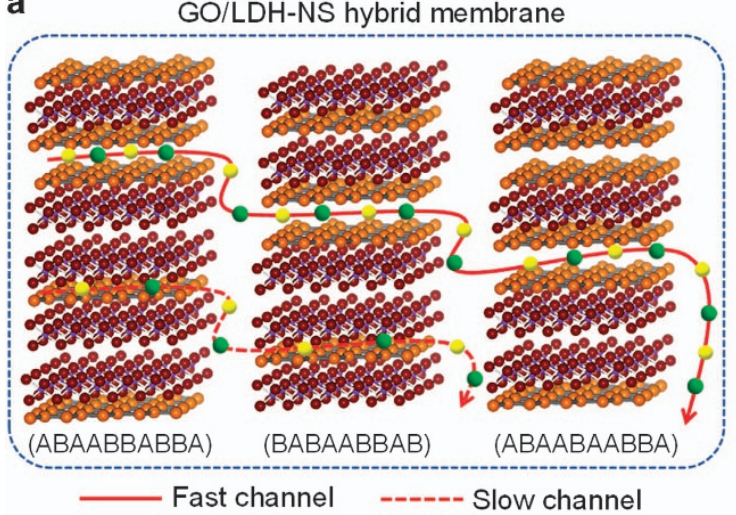

b
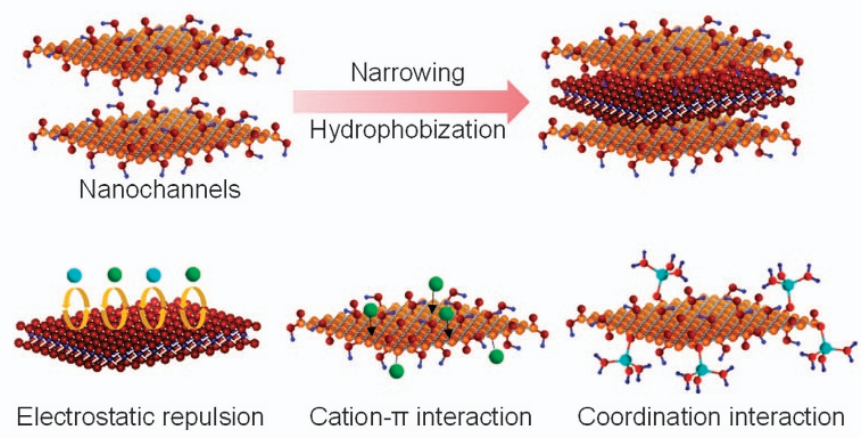

Transition metal cation

Main-group metal cation

Figure 5 (a) Schematic diagram illustrating the possible nanochannels within GO/LDH-NS hybrid membranes. (b) The role of LDH-NS in the modification of GO nanochannels (top panel) and the diverse interactions between various cations and NS (bottom panel). 


\section{Mechanism}

On the basis of the above experimental results, the mechanism for the excellent charge-guided ion filtration and separation performances of the GO/LDH-NS hybrid membranes is discussed as follows. Within the hybrid membrane, numerous superlattice units consisting of anionic GO and cationic LDH-NS with various stacking sequences and compositions are stacked together to form a lamellar structure, as illustrated in Figure 5a. In this structure, two types of interlayer nanochannels are formed mainly for mass transport: small portions of adjacent GO-NS are stacked together to form fast channels, whereas large portions of adjacent GO and LDH-NS are stacked together to form slow channels because of the hydrophobic interfaces of LDH-NS. On the other hand, according to the results in Supplementary Figure S8, ion permeations through LDH-NS multilayer stacks are insignificant, indicating that the nanochannels between adjacent LDH-NS are nearly impermeable because of the presence of two hydrophobic nanochannel walls.

By intercalating LDH-NS into the GO laminates, the pristine $s p^{2}$ nanocapillaries in pure GO membranes are significantly narrowed. This is demonstrated by the change in average interlayer spacing from $\sim 1.35 \mathrm{~nm}$ of the GO membranes in a fully wet state (Figure $2 \mathrm{~d}$ ) to $\sim 0.77 \mathrm{~nm}$ of the GO/LDH-NS hybrid membranes (Figure $2 \mathrm{c}$ ), which extends the steric hindrance effect to the small ion regime, affording a pronounced selectivity to small ions (for example, the effective separation of $\mathrm{Al}^{3+}$ with Stokes and hydrated diameters of 0.88 and $0.95 \mathrm{~nm}$, respectively, from other monovalent and divalent cations, as shown in Figure 4). In addition, the hydrophobic interfaces of LDH-NS induce a strong dehydration effect to hydrated ions, further weakening the electrostatic screening of hydration shells and enhancing the interactions between ions and NS. The ion dehydration effect can be demonstrated by the permeability of all the divalent cations investigated $\left(\mathrm{Mg}^{2+}, \mathrm{Ca}^{2+}, \mathrm{Co}^{2+}\right.$ and $\left.\mathrm{Ni}^{2+}\right)$ with larger hydrated diameters than the average interlayer spacing of GO/LDH-NS hybrid membranes (Supplementary Table S2). The narrowing and hydrophobization of the nanochannels by intercalating LDH-NS are illustrated in Figure 5b (top panel). Within the GO-LDH nanochannels, diverse electrostatic and chemical interactions enhanced by the dehydration of ions are responsible for the excellent charge-guided selective ion transmembrane permeations, as illustrated in Figure $5 \mathrm{~b}$ (bottom panel). For the cations with various valences, the positive charges of LDH-NS induce a stronger electrostatic repulsion to higher charged cations, and the anchoring originating from the cation- $\pi$ interactions between higher charged main-group metal cations and $s p^{2}$ clusters on GO-NS is stronger than that of the lower charged ones. ${ }^{28}$ The synergistic effect of electrostatic repulsions by LDH-NS and cation- $\pi$ captures by GO-NS gives rise to the effective separation of cations with various valences, beyond the predictions from Coulomb's law of discrete charge systems. On the other hand, for transition metal cations (for example, $\mathrm{Co}^{2+}$ and $\mathrm{Ni}^{2+}$ ), the coordination interactions with oxygen functional groups induce an extra strong capture by GO-NS, further lowering the permeations of transition metal cations compared with main-group metal cations with the same charges, ${ }^{28,29}$ as shown in Figures $4 \mathrm{a}, \mathrm{b}$, e and $\mathrm{f}$. For different anions, the electrostatic attractions from cationic LDH-NS and the electrostatic repulsions from anionic GO-NS somewhat counteract each other, leading to the independence of the cation selectivity of the hybrid membranes on anionic species, as shown in Figures $4 \mathrm{c}$ and $\mathrm{d}$.

\section{CONCLUSIONS}

In summary, we have synthesized a novel hybrid membrane consisting of anionic GO and cationic Co-Al (or Mg-Al) LDH-NS superlattice units by a simple vacuum-assisted assembly method. The as-prepared hybrid membranes possess a series of characteristics, such as being area- and thickness-controllable, semitransparent, flexible, freestanding and mechanically robust. The interlayer spacing of the hybrid membrane is insensitive to humidity variations, ensuring the structural stability for solution-based mass transport applications. Typically, the vacuum-assisted processing of the hybrid membrane can be finished within 2-3 min, which is extremely promising for the development of industrial-scale production. The ion permeation properties of the hybrid membranes were systematically investigated in comparison with bare microfilters, GO multilayer stacks, LDH-NS multilayer stacks and their bulk-stratified membranes, indicating that the cations bearing various valences can be effectively separated strictly according to their charges, independent of the cationic and anionic species. The relative selectivity between monovalent and trivalent cations is as high as 30 , which is not achievable by other control membranes. Finally, the mechanism for the excellent charge-guided ion filtration and separation performances of the hybrid membranes was discussed, indicating that the strong narrowing and hydrophobization of the pristine GO nanochannels by the intercalation of LDH-NS and the synergistic effect of electrostatic interactions from LDH-NS and chemical interactions (coordination, cation- $\pi$ interaction) from GO-NS give rise to the effective separation of cations with various valences. These excellent charge-guided ion filtration and separation properties of GO/LDH-NS hybrid membranes make them promising in a series of mass transport applications such as wastewater treatment and reuse, chemical refinement and biomimetic selective ion transport for biological uses.

\section{CONFLICT OF INTEREST}

The authors declare no conflict of interest.

\section{ACKNOWLEDGEMENTS}

This work was supported by the National Program on Key Basic Research Project, China (2011CB013000 and 2014CB932401) and the World Premier International Center Initiative (WPI) on Materials Nanoarchitectonics, MEXT, Japan. Support from JSPS KAKENHI grants (15H03534 and 15K13296) is also acknowledged.

1 Shannon, M. A., Bohn, P. W., Elimelech, M., Georgiadis, J. G., Marinas, B. J. \& Mayes, A. M. Science and technology for water purification in the coming decades. Nature 452, 301-310 (2008)

2 Logan, B. E. \& Elimelech, M. Membrane-based processes for sustainable power generation using water. Nature 488, 313-319 (2012)

3 AghaKouchak, A., Feldman, D., Hoerling, M., Huxman, T. \& Lund, J. Water and climate: recognize anthropogenic drought. Nature 524, 409-411 (2015).

4 Humplik, T., Lee, J., O'Hern, S. C., Fellman, B. A., Baig, M. A., Hassan, S. F., Atieh, M. A., Rahman, F., Laoui, T., Karnik, R. \& Wang, E. N. Nanostructured materials for water desalination. Nanotechnology 22, 292001 (2011).

5 Whitby, M. \& Quirke, N. Fluid flow in carbon nanotubes and nanopipes. Nat. Nanotechnol. 2, 87-94 (2007).

6 Park, H. G. \& Jung, Y. Carbon nanofluidics of rapid water transport for energy applications. Chem. Soc. Rev. 43, 565-576 (2014).

7 Zhao, Y., Xie, Y., Liu, Z., Wang, X., Chai, Y. \& Yan, F. Two-dimensional material membranes: an emerging platform for controllable mass transport applications. Small 22, 4521-4542 (2014).

8 Liu, G., Jin, W. \& Xu, N. Graphene-based membranes. Chem. Soc. Rev. 44, 5016-5030 (2015).

9 Goh, P. S. \& Ismail, A. F. Graphene-based nanomaterial: the state-of-the-art material for cutting edge desalination technology. Desalination 356, 115-128 (2015).

10 Huang, H., Ying, Y. \& Peng, X. Graphene oxide nanosheet: an emerging star material for novel separation membranes. J. Mater. Chem. A 2, 13772-13782 (2014).

11 Hegab, H. M. \& Zou, L. Graphene oxide-assisted membranes: Fabrication and potential applications in desalination and water purification. J. Membr. Sci. 484, 95-106 (2015). 
12 Mahmoud, K. A., Mansoor, B., Mansour, A. \& Khraisheh, M. Functional graphene nanosheets: The next generation membranes for water desalination. Desalination 356, 208-225 (2015).

13 Joshi, R. K., Alwarappan, S., Yoshimura, M., Sahajwalla, V. \& Nishina, Y. Graphene oxide: the new membrane material. Appl. Mater. Today 1, 1-12 (2015).

14 Schniepp, H. C., Li, J. L., McAllister, M. J., Sai, H., Herrera-Alonso, M., Adamson, D. H., Prud'homme, R. K., Car, R., Saville, D. A. \& Aksay, I. A. Functionalized single graphene sheets derived from splitting graphite oxide. J. Phys. Chem. B 110, 8535-8539 (2006).

15 Go'mez-Navarro, C., Weitz, T. R., Bittner, A. M., Scolari, M., Mews, A., Burghard, M. \& Kern, K. Electronic transport properties of individual chemically reduced graphene oxide sheets. Nano Lett. 7, 3499-3503 (2007).

16 Eda, G., Fanchini, G. \& Chhowalla, M. Large-area ultrathin films of reduced graphene oxide as a transparent and flexible electronic material. Nat. Nanotechnol. 3, 270-274 (2008)

17 Mattevi, C., Eda, G., Agnoli, S., Miller, S., Mkhoyan, K. A., Celik, O., Mastrogiovanni, D., Granozzi, G., Garfunkel, E. \& Chhowalla, M. Evolution of electrical, chemical, and structural properties of transparent and conducting chemically derived graphene thin films. Adv. Funct. Mater. 19, 2577-2583 (2009).

18 Robinson, J. T., Zalalutdinov, M., Baldwin, J. W., Snow, E. S., Wei, Z., Sheehan, P. \& Houston, B. H. Wafer-scale reduced graphene oxide films for nanomechanical devices. Nano Lett. 8, 3441-3445 (2008).

19 Robinson, J. T., Perkins, F. K., Snow, E. S., Wei, Z. \& Sheehan, P. E. Reduced graphene oxide molecular sensors. Nano Lett. 8, 3137-3140 (2008).

$20 \mathrm{Eda}$, G. \& Chhowalla, M. Chemically derived graphene oxide: towards large-area thin-film electronics and optoelectronics. Adv. Mater. 22, 2392-2415 (2010).

21 Loh, K. P., Bao, Q., Eda, G. \& Chhowalla, M. Graphene oxide as a chemically tunable platform for optical applications. Nat. Chem. 2, 1015-1024 (2010).

22 Nair, N. N., Wu, H. A., Jayaram, P. N., Grigorieva, I. V. \& Geim, A. K. Unimpeded permeation of water through helium-leak-tight graphene-based membranes. Science 335, 442-444 (2012).

23 Joshi, R. K., Carbone, P., Wang, F. C., Kravets, V. G., Su, Y., Grigorieva, I. V., Wu, H. A., Geim, A. K. \& Nair, R. R. Precise and ultrafast molecular sieving through graphene oxide membranes. Science 343, 752-754 (2014).

$24 \mathrm{Han}$, Y., Xu, Z. \& Gao, C. Ultrathin graphene nanofiltration membrane for water purification. Adv. Funct. Mater. 23, 3693-3700 (2013).

$25 \mathrm{Hu}, \mathrm{M}$. \& Mi, B. Enabling graphene oxide nanosheets as water separation membranes. Environ. Sci. Technol. 47, 3715-3723 (2013)

26 Huang, H., Mao, Y., Ying, Y., Liu, Y., Sun, L. \& Peng, X. Salt concentration, pH and pressure controlled separation of small molecules through lamellar graphene oxide membranes. Chem. Commun. 49, 5963-5965 (2013).

27 Sun, P., Zhu, M., Wang, K., Zhong, M., Wei, J., Wu, D., Xu, Z. \& Zhu, H. Selective ion penetration of graphene oxide membranes. ACS Nano 7, 428-437 (2013).

28 Sun, P., Zheng, F., Zhu, M., Song, Z., Wang, K., Zhong, M., Wu, D., Little, R. B., Xu, Z. \& Zhu, H. Selective trans-membrane transport of alkali and alkaline earth cations through graphene oxide membranes based on cation- $\pi$ interactions. ACS Nano 8, 850-859 (2014)

29 Sun, P., Liu, H., Wang, K., Zhong, M., Wu, D. \& Zhu, H. Selective ion transport through functionalized graphene membranes based on delicate ion-graphene interactions. J. Phys. Chem. C 118, 19396-19401 (2014).

30 Huang, H., Song, Z., Wei, N., Shi, L., Mao, Y., Ying, Y., Sun, L., Xu, Z. \& Peng, X. Ultrafast viscous water flow through nanostrand-channelled graphene oxide membranes. Nat. Commun. 4, 2979 (2014).

31 Gao, S. J., Qin, H., Liu, P. \& Jin, J. SWCNT-intercalated GO ultrathin films for ultrafast separation of molecules. J. Mater. Chem. A 3, 6649-6654 (2015).

32 Han, Y., Jiang, Y. \& Gao, C. High-flux graphene oxide nanofiltration membrane intercalated by carbon nanotubes. ACS Appl. Mater. Interfaces 7, 8147-8155 (2015).

33 Sun, P., Chen, Q., Li, X., Liu, H., Wang, K., Zhong, M., Wei, J., Wu, D., Ma, R., Sasaki, T. \& Zhu, H. Highly efficient quasi-static water desalination using monolayer graphene oxide/titania hybrid laminates. NPG Asia Mater 7, e162 (2015).
34 Allmann, R. The crystal structure of pyroaurite. Acta Crystallogr. B 24, 972-977 (1968).

35 Braterman, P. S., Xu, Z. P., Yarberry, F. in Handbook of Layered Materials (eds Auerbach S. M., Carrado K. A. \& Dutta P. K.) 373-474 (Marcel Dekker, Inc., New York, NY, USA, 2004).

36 Rives, V. Layered Double Hydroxides: Present and Future (Nova Science Publishers, Inc., New York, NY, USA, 2006).

37 Zhao, Y., Chen, G., Bian, T., Zhou, C., Waterhouse, G. I. N., Wu, L.-Z., Tung, C. H., Smith, L. J., O'Hare, D. \& Zhang, T. Defect-rich ultrathin ZnAl-layered double hydroxide nanosheets for efficient photoreduction of $\mathrm{CO}_{2}$ to $\mathrm{CO}$ with water. Adv. Mater. 27, 7824-7831 (2015)

38 Zhao, Y., He, S., Wei, M., Evans, D. G. \& Duan, X. Hierarchical films of layered double hydroxides by using a sol-gel process and their high adaptability in water treatment. Chem. Commun. 46, 3031-3033 (2010).

39 Hibino, T. \& Jones, W. New approach to the delamination of layered double hydroxides. J. Mater. Chem. 11, 1321-1323 (2001).

40 Li, L., Ma, R., Ebina, Y., Iyi, N. \& Sasaki, T. Positively charged nanosheets derived via total delamination of layered double hydroxides. Chem. Mater. 17, 4386-4391 (2005).

41 Liu, Z., Ma, R., Osada, M., Iyi, N., Ebina, Y., Takada, K. \& Sasaki, T. Synthesis, anion exchange, and delamination of Co-Al layered double hydroxide: assembly of the exfoliated nanosheet/polyanion composite films and magneto-optical studies. J. Am. Chem. Soc. 128, 4872-4880 (2006).

42 Liang, J., Ma, R., Iyi, N., Ebina, Y., Takada, K. \& Sasaki, T. Topochemical synthesis, anion exchange, and exfoliation of Co-Ni layered double hydroxides: A route to positively charged Co-Ni hydroxide nanosheets with tunable composition. Chem. Mater. 22, 371-378 (2010).

$43 \mathrm{Ma}, \mathrm{R}$. \& Sasaki, T. Nanosheets of oxides and hydroxides: Ultimate 2D charge-bearing functional crystallites. Adv. Mater. 22, 5082-5104 (2010).

44 Wang, Q. \& O'Hare, D. Recent advances in the synthesis and application of layered double hydroxide (LDH) nanosheets. Chem. Rev. 112, 4124-4155 (2012).

$45 \mathrm{Ma}, \mathrm{R}$. \& Sasaki, T. Two-dimensional oxide and hydroxide nanosheets: controllable high-quality exfoliation, molecular assembly, and exploration of functionality. Acc. Chem. Res. 48, 136-143 (2015).

$46 \mathrm{Ma}$, R., Liu, X., Liang, J., Bando, Y. \& Sasaki, T. Molecular-scale heteroassembly of redoxable hydroxide nanosheets and conductive graphene into superlattice composites for high-performance supercapacitors. Adv. Mater. 26, 4173-4178 (2014).

47 Ma, W., Ma, R., Wang, C., Liang, J., Liu, X., Zhou, K. \& Sasaki, T. A superlattice of alternately stacked $\mathrm{Ni}-\mathrm{Fe}$ hydroxide nanosheets and graphene for efficient splitting of water. ACS Nano 9, 1977-1984 (2015).

48 Hummers, W. S. Jr \& Offeman, R. E. Preparation of graphitic oxide. J. Am. Chem. Soc. 80, 1339 (1958).

49 Park, S. \& Ruoff, R. S. Chemical methods for the production of graphenes. Nat. Nanotechnol. 4, 217-224 (2009).

50 Lerf, A., Buchsteiner, A., Pieper, J., Schottl, S., Dekany, I., Szabo, T. \& Boehm, H. P. Hydration behavior and dynamics of water molecules in graphite oxide. J. Phys. Chem. Solids 67, 1106-1110 (2006).

(c) (i) This work is licensed under a Creative Commons Attribution 4.0 International License. The images or other third party material in this article are included in the article's Creative Commons license, unless indicated otherwise in the credit line; if the material is not included under the Creative Commons license, users will need to obtain permission from the license holder to reproduce the material. To view a copy of this license, visit http:// creativecommons.org/licenses/by/4.0/

Supplementary Information accompanies the paper on the NPG Asia Materials website (http://www.nature.com/am) 